\title{
ÉCHANGE DE RESSOURCES
}

\section{GUIDE DE PARRAINAGE}

Un guide complet et à jour sur le parrainage des réfugiés au Canada vient de paraître. II est le fruit des efforts concertés de nombreux organismes bénévoles et gouvernementaux et a été conçu en fonction des réfugiés de diverses origines ethniques qui s'établissent dans différentes parties du Canada. II offre des renseignements pratiques sur la façon de constituer un groupe de parrainage, les aspects financiers du parrainage, les rapports entre le répondant et le réfugié, la façon d'aider les réfugiés à trouver du travail au Canada et bien d'autres aspects encore. En commandant le guide, précisez l'origine ethnique du réfugié dont vous vous occupez ou que vous comptez aider, ainsi que la région du Canada où le réfugié sera parrainé. Des monographies sur l'Argentine, le Cambodge, le Chili, le Laos, l'Uruguay et le Vietnam sont actuellement disponibles. Des bottins faisant état des services locaux sont également publiés pour chaque province, à l'exception du Québec qui a son propre ministère de l'Immigration et dont les procédures et les publications de parrainage diffèrent et s'adressent aux répondants de cette province. Une contribution facultative de $\$ 5$ serait appréciée pour couvrir les frais d'impression. Ce guide est distribué par:

La Fondation canadienne des réfugiés

C.P. 50000

Ottawa (Ontario)

K1N 9 G9

\section{TROUSSE ÉDUCATIVE POUR LES ÉLÈVES DU SECONDAIRE}

"The World's Homeless: Should We Help Them?" est une trousse éducative conçue pour aider les élèves à se sensibiliser aux questions touchant les réfugiés. Elle peut être utilisée dans les disciplines suivantes: sciences sociales, histoire, actualités et multiculturalisme. La trousse comprend:

- trente journaux composés de divers articles et éditoriaux concernant les réfugiés:

- une affiche; et

- un guide complet conçu pour aider les enseignants à structurer des cours à partir de ces documents.

Coût: $\$ 10$

Contact: L.A. Weigl Educational Associates, Ltd. 2114 College Ave.

Regina, Saskatchewan S4P 1C5

\section{TROUSSE ÉDUCATIVE POUR LES ENFANTS}

"The Reflugee Experience - Perspectives on Refugee issues" est une trousse éducative multi-média conçue à l'intention des enfants de 9 à 14 ans pour aider ces derniers à mieux comprendre les problèmes reliés aux réfugiés. Elle peut être utilisée en milieux scolaire, paroissial ou communautaire. La trousse permet aux élèves de se familiariser avec le concept des réfugiés, de se pencher sur les causes fondamentales de leur déplacements, d'explorer la politique du gouvernement canadien relative aux réfugiés et d'examiner les conditions d'admissibilité; enfin, les élèves prennent connaissance des difficultés reliées au réétablissement des réfugiés une fois leur cas approuvé. La trousse comprend aussi de la documentation détaillée sur la situation des réfugiés dans le Sud-est asiatique (Laos, Kampuchea, Vietnam), en Amérique latine (Chili), et en Afrique (Corne d'Afrique, Zimbabwe)

La trousse permet en outre d'aborder le sujet de différentes façons, en utilisant des jeux de simulation, des disques, des bandes magnétiques, des livres, des affiches, des contes ou des discussions de groupe.

Elle comprend un guide de l'usager, 20 livres, 20 cartes géographiques, 10 jeux sur table, 1 disque, 2 séries de diapositives, 1 film avec bande sonore, 1 chemise contenant de la documentation historique, 1 cartable de matériel pédagogique et un jeu de simulation.

- Coût: $\$ 40.00$

Contact: Global Community Centre

Box One Million

94 Queen Street South

Kitchener, Ont.

N2G 1 V9

\section{CONFÉRENCES}

\section{COMITÉ PERMANENT DES ORGAN- ISMES CANADIENS AU SERVICE DES RÉFUGIÉS}

\author{
Rencontre Annuelle \\ 27,28 et 29 novembre 1981 \\ 6:00 vendredi - 4:00 dimanche \\ Centre Marial Montfortain \\ Montréal
}

Pour plus de renseignements, communiquer avec le Comité Permanent des Organismes Canadiens au Service des Réfugiés, 40 St. Clair Ave. East, Suite 201, Toronto, Ontario M4T $1 \mathrm{M} 9$

\section{LE POINT SUR LA SITUATION MONDIALE}

Halifax

le 28 novembre 1981

Une conférence d'une journée sur les réfugiés aura lieu à compter de 9h au YMCA de Halifax, située au 1565, rue South Park. Cette conférence est offerte conjointement par I'International Education Centre de St. Mary's University, le Metropolitain Immigrant Services Association et le YMCA.

Les sujets traités par les conférenciers porteront sur la situation mondiale des réfugiés, les problèmes régionaux, la politique du gouvernement canadien sur les réfugiés et la situation des réfugiés au niveau local.

Frais d'inscription: $\$ 5.00$

Pour de plus amples renseignements, contacter Erla McClelland, YMCA, (902) 422-6437. 